\title{
Efectividad de una pauta de corticoides vía general para disminuir las recaídas del crup laríngeo
}

\author{
F. J. Ortiz leal, Mạ A. Palma Suárez, A. J. Belmonte Rodríguez, \\ M. A. M ORENO García*
}

Residente de Medicina Familiar y Comunitaria en el Centro de Salud de Carranque.

Unidad Docente de MF y C de Málaga. * M édico de Familia en el Centro de Salud de

Carranque. Unidad Docente de MF y C de Málaga

\section{RESUMEN}

La utilización de monodosis de corticoides ora les en el tratamiento al alta del crup leve-moderado en niños es una práctica frecuente en nuestro medio. Nos hemos preguntado si dicha actuación disminuye las recaidas del cuadro. Esta cuestión no se resuelve con la consulta de los textos básicos de pediatría, por lo que decidimos utilizar la meto dología de la medicina basada en las pruebas con la que seleccionamos y criticamos un ensayo clíni co donde se valora la administración de una única dosis de dexametasona en niños con crup leve-mo derado cuando son dados de alta.

Palabras clave: Crup. Glucocorticoides. Dexa metasona. Tratamiento farmacológico.
Effectiveness of small oral dose of steroids to de crease the ongoing croup

\begin{abstract}
It's a frequent practice in our environment to use a small single oral dose of steroids for the treatment of mild croup in children. We have asked ourselves if this action decrease the ongoing croup. This question is not solved with the paediatric texts, then we decided to use the evidence based medicine methodology. We analysed a clinical trial that asses the administration of a small single oral dose of dexamethasone in children with mild croup.
\end{abstract}

Key words: Croup. Glucocorticoids. Dexame thasone. Drug therapy. Drug utilitation review.

\section{DESCRIPCIÓN DEL CASO CLÍNICO}

Varón de 3 años de edad que acude a la urgencia de nuestro Centro de Salud por la aparición brusca de afonía, tos metálica y estridor inspiratorio.

Sin antecedentes personales de enfermedad, ni ingresos hospitalarios previos. No toma ningún tratamiento actualmente. Desde hace 48 horas presenta un cuadro de infección de vías respiratorias altas que ha recibido tratamiento sintomático con antitusígenos (Cloperastina) por su pediatra, no presentando afectación de vías aéreas inferiores.

Buen estado general, buen nivel de consciencia, coloración de piel normal, intranquilo aunque no presenta agitación. Exploración de cabeza y cuello normal. Discreta dificultad respiratoria con leve tiraje supraesternal y auscultación cardiorrespiratoria normal. Abdomen: normal. Extremidades normales. ORL: orofaringe discretamente hiperémica, sin placas ni exudados. Otoscopia bilateral normal.

Se diagnostica clínicamente de laringitis aguda ligera-moderada, según la puntuación obtenida en el Score-Taussing (Tabla I). Se le administra, como tratamiento de urgencias, aerosoles con suero fisiológico y budesonida $2 \mathrm{mg}$. Tras tratamiento el niño mejora notablemente, presentando sólo algún episodio de tos perruna. Es dado de alta y se le pauta tratamiento con humidificación del ambiente y unidosis de corticoide oral (dexametasona). 
Tabla I

VALORACIÓN CLÍNICA: SCORE-TAUSSING*

\begin{tabular}{|c|c|c|c|c|}
\hline & 0 & 1 & 2 & 3 \\
\hline Estridor & No & Leve & Moderado en reposo & $\begin{array}{l}\text { Grave en inspiración y } \\
\text { espiración o ninguno con } \\
\text { disminución intensa de la } \\
\text { entrada de aire }\end{array}$ \\
\hline Retracción & No & Leve & Moderada & $\begin{array}{l}\text { Grave, empleo } \\
\text { considerable de músculos } \\
\text { accesorios }\end{array}$ \\
\hline Entrada de aire & Normal & Disminución leve & Disminución moderada & Disminución grave \\
\hline Color & Normal (0 p) & Normal (0 p) & (Normal 0 p) & Cianótico \\
\hline $\begin{array}{l}\text { Nivel de } \\
\text { conciencia }\end{array}$ & Normal & $\begin{array}{l}\text { Intranquilo si es } \\
\text { importunado }\end{array}$ & $\begin{array}{l}\text { Ansioso, agitado, } \\
\text { intranquilo sin ser } \\
\text { importunado }\end{array}$ & Letárgico, deprimido \\
\hline
\end{tabular}

* Procedencia: cita 1.

\section{SELECCIÓN DEL PROBLEMA}

La variabilidad del abordaje terapéutico en nuestro medio de la laringitis aguda hace que nos preguntemos sobre el beneficio añadido de la corticoterapia oral al alta, frente a sólo las medidas generales habituales (ventilación y humidificación ambiental) para disminuir el número de recaídas.

\section{FORMULACIÓN DE LA PREGUNTA}

En un paciente menor de 10 años con laringitis leve-moderada una pauta corta de corticoides orales (1 a 3 días) al alta domiciliaria, ¿disminuirá la probabilidad de recaída del crup?

\section{FUENTES DE INFORMACIÓN Y ESTRATEGIA DE BÚSQUEDA}

Para aclarar esta duda acudimos a los textos básicos de Pediatría ${ }^{1-3}$, en los que no encontramos datos concluyentes que dieran respuesta a nuestra pregunta.

En la Cochrane Library encontramos una revisión "Glucocorticoids for croup" que tampoco contestaba nuestra pregunta.

Se realizó una búsqueda bibliográfica en Medline a través del portal gratuito de Grateful Med. Introduciendo como términos principales del
MESH laryngitis/drug therapy OR, laryngitis/pre vention and control, y obtuvimos 283 artículos. Utilizamos una serie de términos limitadores para acotar la búsqueda: a) que se tratasen de ensayos clínicos randomizados; b) que se realizasen en humanos; c) en un rango de edad comprendido entre 0 y 18 años; y d) publicados entre 1990 y 2000. De este modo obtuvimos 28 artículos. De todos ellos únicamente cuatro artículos se ajustaban a las características del caso clínico presentado, siendo el resto descartados por tratarse de casos distintos ya que unos incluían variantes como la infección, otros lo que hacían era comparar distintas dosis de dexametasona y otros estudiaban los corticoides de forma aerosolizada. Dos de los cuatro artículos mencionados fueron rechazados por tratarse de pacientes hospitalizados en los que se analizaba la efectividad de dicho tratamiento; el tercero de los artículos se trataba de una citación del artículo elegido ${ }^{5}$. El artículo más adecuado tenía como título, "Efficacy of a small single dose of oral dexamethasone for outpatient croup: a double blind placebo controlled clinical trial".6. Se trata de un ensayo clínico randomizado a doble ciego que valora la efectividad de una dosis pequeña de dexametasona oral $(0,15 \mathrm{mg} / \mathrm{kg})$ en pacientes no hospitalizados con crup. Utilizan 100 pacientes comprendidos entre los 4 y 122 meses a los que se divide aleatoriamente en dos grupos similares y se les administra tratamiento con dexametasona o placebo. Los criterios de inclusión fueron niños sin problemas médicos agu- 


\begin{tabular}{|c|c|c|c|c|}
\hline \multicolumn{5}{|c|}{ VALORACIÓN DE LA SEVERIDAD DEL CRUP QUE MANEJA EL ARTÍCULO DE REFERENCIA* } \\
\hline & 0 & 1 & 2 & 3 \\
\hline Estridor & No & $\begin{array}{l}\text { Leve. Sólo con } \\
\text { esfuerzo de llanto }\end{array}$ & Moderado en reposo & Severo. Bifásico \\
\hline Retracción & No & $\begin{array}{l}\text { Leve. Sólo con } \\
\text { esfuerzo de llanto }\end{array}$ & Moderada en reposo & Severo. Bifásico \\
\hline
\end{tabular}

dos o crónicos, que acudieron a un servicio de urgencias hospitalario y que se diagnosticaron de crup, no lo suficientemente severo como para ser ingresados. La severidad del crup la baremaron mediante una escala (Tabla II) similar a la que exponemos en nuestro caso clínico. No valoraron en dicha escala la cianosis para evitar la variabilidad subjetiva en la apreciación, monotorizando a los niños con pulsioximetría. Excluyeron aquéllos que no tenían teléfono o por dificultad con el idioma (inglés); que hubiesen recibido en la semanas precedentes corticoides; que tuviesen alguna predisposición a padecer afectación de vías aéreas superiores; que presentaran historia de estridor prolongado, o que diesen la impresión clínica de padecer algo más que un crup. Se contacta telefónicamente a los 7-10 días con sus domicilios familiares. Los resultados se miden en la necesidad de asistencia médica por recaída del crup. A lo largo del seguimiento se pierden 4 pacientes.

Se analizó dicho artículo siguiendo la guía de epidemiología clínica de Sackett publicada en JA-

\begin{tabular}{|c|c|c|}
\hline \multicolumn{3}{|c|}{ Tabla III } \\
\hline \multicolumn{3}{|c|}{ SEVERIDAD DEL CRUP } \\
\hline & Corticoides orales & No corticoides orales \\
\hline Recaídas & 2 & 8 \\
\hline No recaídas & 48 & 42 \\
\hline \multicolumn{3}{|c|}{$\begin{array}{l}\text { Riesgo relativo } 0,25 \text {, intervalo de confianza al } 95 \% \text { [0,06- } 1,12] \\
\text { Reducción de riesgo absoluto } 12 \% \text {, intervalo de confianza al } 95 \% \\
{[0,4-23,5 \%] \text {. }} \\
\text { Reducción de riesgo relativo } 75 \% \text {, intervalo de confianza al } 95 \% \\
\text { [-12-94\%]. Número necesario a tratar (NNT) } 8 \% \text {, intervalo de } \\
\text { confianza al } 95 \%[4-209] \text {. }\end{array}$} \\
\hline
\end{tabular}

MA7 comprobando que cumplía los criterios de validación, así como que los resultados eran aplicables a nuestro caso. Nos pusimos en el más desfavorable de los casos ya que incluimos a los 4 pacientes que se perdían en el estudio en el grupo de los que recaían con corticoides o de los que no recaían con placebo y analizando estos resultados se obtuvieron los siguientes parámetros aplicando un intervalo de confianza al 95\% (Tabla III):

Riesgo relativo (RR) 0,25 (0,06-1,12); reducción de riesgo absoluto (RRA) $12 \%(0,4-23,5 \%)$; reducción de riesgo relativo (RRR) 75\% (-12-94\%); número necesario a tratar (NNT) 8.

\section{RECOMENDACIONES PARA LA PRÁCTICA}

Según los resultados obtenidos tras la crítica del artículo se puede afirmar que las conclusiones no son suficientemente evidentes como para aplicar esta medida terapéutica en la práctica habitual, pues con un intervalo de confianza al $95 \%$ la variabilidad para obtener resultados positivos es muy amplia. Además, el estudio no valora los posibles efectos adversos que dicha práctica provocaría.

Sería necesario, por lo tanto, nuevos ensayos con una población de estudio mayor en el que se evaluaran los posibles efectos secundarios de esta medida terapéutica.

\section{CORRESPONDENCIA:}

Francisco José Ortíz Leal

Centro de Salud de Carranque

C/ Virgen Inmaculada, 2

Málaga

Tel. 95270700; 952610500 


\section{Bibliografía}

1. Benéitez Maestre AM, Calvo Rey C, Del Castillo Martín F. Dificultad respiratoria alta. Crup y epiglotitis. En: Zafra M

A, Calvo C, Garcia ML, Baquero F, Arribas N, Jiménez J, et al. Manual de diagnóstico y terapéutica en pediatría. $3^{\mathrm{a}}$ ed. Madrid: Publires, 1996; 479-81.

2. Steven M, Selbst MD. Infección de las vías aéreas superiores. En: Schwartz MW, Chaney EB, Curry TA, Ludwig S. Manual de atención Primaria en pediatría. $2^{\mathrm{a}}$ ed. Madrid: Mosby, 1994; 495-501.

3. Pastor X. Infecciones laringo-bronquiales. Cruz M. Tratado Pediatría. $7^{a}$ ed. Barcelona: Espax, 1993; 1300-8.

4. Ausejo M, Saenz A, Pham B, Kellner JD, Johnson DW,
Moher D, et al. Glucocorticoids for croup (Cochrane Review). In: The Cochrane Library, Issue 2, 2001; Oxford. Update software.

5. Slawson JG. Efficacy of oral dexamethasone for outpatient croup. J Fam Pract 1996; 43: 436.

6. Geelhoed GC, Turner J, Macdonald WB. Efficacy of a small single dose of oral dexamethasone for outpatient croup: a double blind placebo controlled clinical trial. BMJ 1996; 313: 140-2.

7. Evidence Based Medicine Working group. La Medicina Basada en la Evidencia. Guía usuaria de la literatura médica. JAMA ed. Española (Barcelona), 1997. 